\title{
The Role of Astrocytes in the Cause of Alzheimer's Disease
}

\author{
Nadia Priyam ${ }^{1}$ and Andrew Savoy\# \\ ${ }^{1}$ Mount Pisgah Christian School, Johns Creek, GA, USA \\ \#Advisor
}

\section{$\underline{\text { ABSTRACT }}$}

There are three leading hypotheses about the cause of Alzheimer's Disease (AD): the cholinergic theory, where there is a loss of cholinergic neurons; the amyloid hypothesis, where there is an abnormal buildup of amyloid plaques; and the neurotrophic unbalance hypothesis, which states that AD-related loss of cholinergic signaling and altered amyloid precursor protein (APP) processing are due to alterations in nerve growth factor (NGF). This would ultimately mean that the loss of cholinergic neurons and a buildup of amyloid plaques are due to NGF alterations. Astrocytes are involved in the production of amyloid beta, inflammation responses, and nerve growth. Therefore, astrocytes are an essential component of all three AD hypotheses. This paper will discuss various known and hypothesized ways that astrocytes affect the symptoms and possible causes of AD.

\section{Introduction: Alzheimer's Disease}

Alzheimer's disease (AD) is a progressive neurodegenerative condition. People diagnosed with AD gradually lose memory and cognitive abilities. The cause of this disease could be genetic, but there may also be lifestyle etiological factors. There are three stages in AD: mild (early), moderate (middle), and severe (late). In the mild stage, symptoms include forgetting names and misplacing things. In the moderate stage, symptoms become more pronounced and can include forgetting personal experiences, moodiness, and confusion. The severe stage is the most relentless; symptoms include the degradation of communication, physical abilities, and awareness of surroundings.

An astrocyte is a type of glial cell located in the central nervous system (CNS). Its functions include metabolic support to neurons via neurovascular coupling; reaction to injury in the brain; support of essential synaptic functions; and removing waste products. One of these key waste products is amyloid plaque. Shown in figure 1, the astrocyte, A1, is activated and clears the debris in the N1 neuron. Without astrocytes the accumulation of amyloid plaque destroys neuronal connections, which contributes to the progression of AD. Therefore, astrocytes play a significant role in $\mathrm{AD}$. 


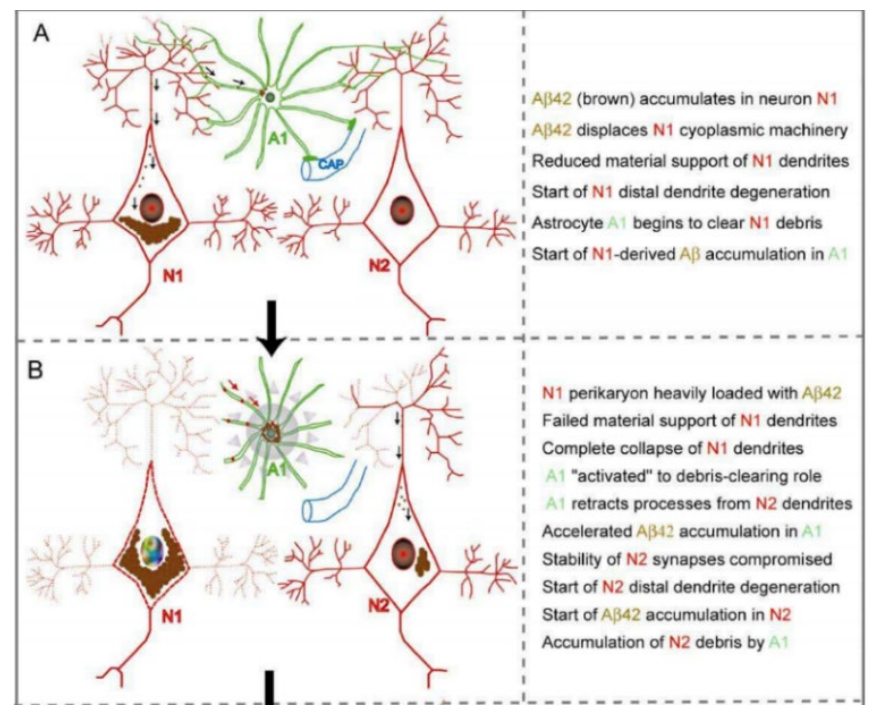

Figure 1: Role of Astrocytes in Amyloid Plaque formation

\section{Amyloid-Beta}

Amyloid beta $(A \beta)$ is a mixture of peptides formed by the amyloid precursor protein (APP). The APP binds to the surface to help direct the movement of neurons. In addition, the APP is eventually cut by an enzyme called, protease and cuts the protein into peptides (figure 2). However, some of these fragments can end up in extracellular spaces in the brain and the accumulation of the $\mathrm{A} \beta$ peptides in the brain forms amyloid plaque. Clumps of amyloid plaque can sever connections in the brain which can lead to memory loss or in other words AD. AD's leading hypothesis is the amyloid hypothesis, where there is an abnormal buildup of amyloid plaques. APP can be processed through different pathways. Abnormal buildup of plaques can surround neurons in the brain and destroy the synaptic connections formed by neurons. As Alzheimer's disease progresses, amyloid plaques destroy neural communication, thus accelerating symptoms. The amyloid theory does not explain all the occurrences and causes of plaque buildup. Many studies have shown that glial cells are involved in amyloid plaque and AD. Astrocytes are the most prominent type of glial cell. They regulate homeostasis in the CNS and contribute to removing amyloid-beta, unlike other glial cells. Additionally, the total number of astrocytes decreases as humans age (Verkhratsky et al., 2010), ${ }^{9}$ explaining how A $\beta$ accumulates progressively as humans age in $\mathrm{AD}$. At the later stages of $\mathrm{AD}$, astrocytes become activated and contribute to neurodegeneration's neuroinflammatory component (Verkhratsky et al., 2010). ${ }^{9}$ When A $\beta$ levels are high, astrocytes engulf massive amounts of $\mathrm{A} \beta$ and only partly digest it, eventually leading to astrocytic defects and neuronal apoptosis (Fakhoury et al., 2018). ${ }^{2}$ These defects can lead to a deficiency in the disposal of amyloid plaques. Defective astrocytes can also be caused by an abnormal buildup of astrocytes due to AB-mediated damage to neurons in the CNS. Additionally, isolated plaques lead to reactive astrogliosis in which there is an abnormal increase of astrocytes. This abnormal buildup can also be caused by infection, autoimmune responses, and other neurodegenerative diseases. Astrogliosis interferes with the regular functions of astrocytes. For example, astrogliosis can cause astrocytic dysfunction causing a buildup of amyloid plaque. Additionally, despite being effective in mediating the degradation of amyloid plaques, astrocytes also produce $\mathrm{A} \beta$ under certain inflammatory conditions (Fakhoury et al., 2018). ${ }^{2}$ For instance, nerve growth factor (NGF), which is released during inflammatory responses, can trigger specific enzymes that drive amyloid-beta production. The reason for the NGF trigger could be an increase of neurotoxicity or a change in the cellular environment. Even though astrocytes clear up amyloid-beta, they can also produce more of it, causing plaques to form. 


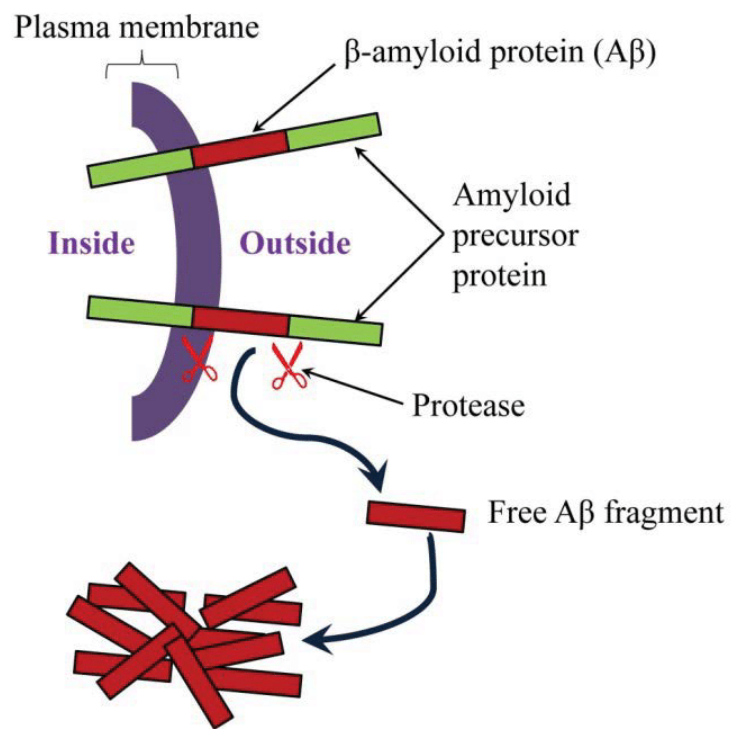

Accumulation of $\mathrm{A} \beta$, plaque formation in extracellular space

Figure 2: Formation of Amyloid Plaque

\section{Neuroinflammation}

Neuroinflammation is a physiological defensive reaction. The functions of neuroinflammation include microorganism defense, tissue repair, and cellular debris elimination. Though neuroinflammation occurs innately as a defensive mechanism when an injury is present in the CNS, alteration in some of this reaction's components can damage the cellular microenvironment and become toxic to the brain. The existence of pathological neuroinflammation has been linked with several neurodegenerative disorders, such as AD (González-Reyes et al., 2017). ${ }^{3}$

When persistent inflammation happens in the brain, microglia, another glial cell, struggles to remove waste. Then astrocytes respond to irritating microglia, which causes the neuron to lose its ability to communicate (Fakhoury et al., 2018). ${ }^{2}$ Therefore, the connections between neurons die. According to González-Reyes, et al. (2017), A $\beta$ 's association with astrocytes causes neuroinflammation and even astrogliosis- the overproduction of astrocytes. ${ }^{3}$ Astrogliosis has also been related to symptoms such as memory loss and trouble with cognitive function. In addition, astrocytes respond to the presence of pro-inflammatory cytokines, which increases the development of $\mathrm{A} \beta$ in reaction (González-Reyes et al., 2017). ${ }^{3}$ Hence the existence of neuroinflammation will increase the development of A $\beta$.

Furthermore, neuroinflammation is linked to cognitive functions in AD. Ianov, et al. (2017) report differences in the gene expression profile of hippocampal subregions and their association with aging and cognition. Specifically, the most important hippocampal regions in memory, CA1, CA3, and the dentate gyrus were isolated. This study found that in a memory task the tested animals were linked to synaptic function in the CA1 region. This proved that these subregions could identify its boundaries in aging and cognition. Additionally, in the specific hippocampus regions, neuroinflammation genes are either expressed at high or low levels and a decline of these genes leads to a decline in $\mathrm{Ca}^{2+}$. And further in the study a decline in $\mathrm{Ca}^{2+}$ was found in the impaired animals that were tested in memory (Ianov, et al. 2017). This decrease in genes expressed can be shown in figure 3. This suggests that these lowly expressed hippocampus regions could be the cause of memory loss in the first stages of AD.

These findings indicate that neuroinflammation may arise at the early stages of the disease, or that neuroinflammation could be responsible for the presence of abnormal development and aggregation of A $\beta$. Overall, astrocytes tend to be deeply involved in inflammatory responses in AD. 


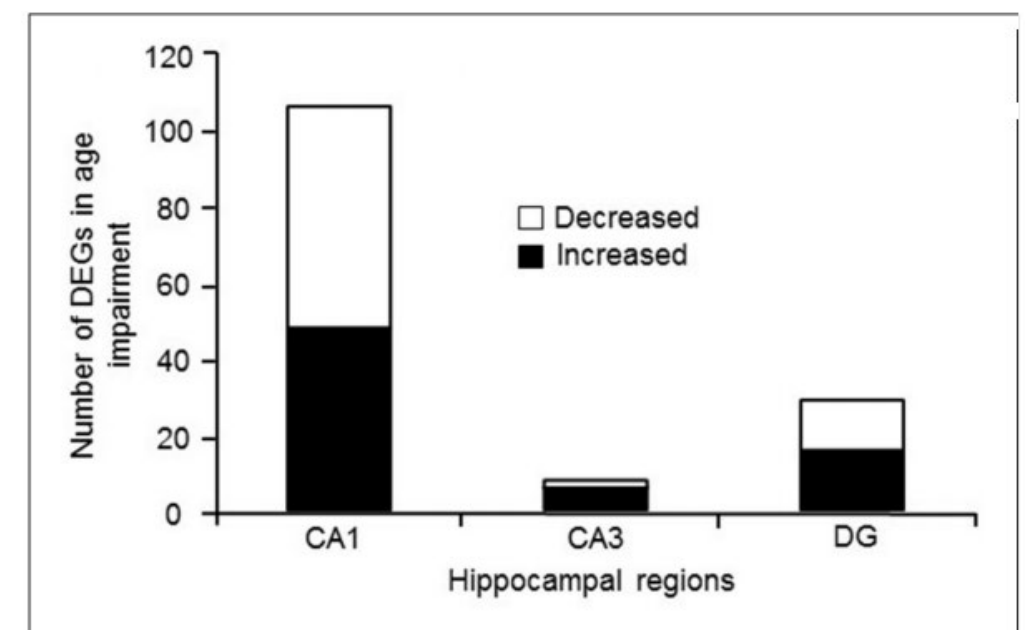

Figure 3: Number of genes expressed in CA1, CA3, and DG hippocampal regions

\section{Growth Factors}

Growth factors (GF) are naturally occurring proteins in the brain and body involved in many cell processes such as survival and division. There are many growth factors, including neurotrophins such as nerve growth factor (NGF) and brain-derived neurotrophic factor (BDNF), as well as transforming growth factor-beta (TGF-B). The differentiation of these growth factors is based on the growth factors' function and the receptor(s) they bind to.

NGF is a neurotrophic factor that is important in the growth and the support of neurons. NGF is also crucial for the survival of neurons. NGFs bind to different receptors that carry out different functions. For example, the NGF binds to the NTRK1 receptor, which then is used to differentiate neurons and avoid cell death. BDNF is essential for learning and memory. It is primarily found in the hippocampus. Studies have shown that BDNF has a negative correlation with amyloid plaque (Tanila, 2017), ${ }^{8}$ but it is a possible approach to the treatment of AD. In the study of (Tanila, 2017), researchers crossbred mice overexpressed APP mice with BDNF mice. They found that the mice with only half of the BDNF gene had impaired spatial learning.

Additionally, TGF is important for roles in cell regulation and immunity. TGF-B induces APP, which plays a key role in $\mathrm{AD}$ and the production of amyloid-beta.

\section{Over- and Under-expression of Growth Factors}

The overexpression of the anti-inflammatory cytokine transforming growth factor-B1 (TGF-B1) in transgenic mice induced higher expression of APP isoforms and increased $A \beta$ generation in cerebral tissues. Furthermore, exogenous TGF-B1 enhances APP synthesis in astrocytes and leads to $A \beta$ generation.

Under expression of NGF in old age has been shown to lead to AD-like pathology (Houeland et al., 2010). ${ }^{4}$ In the (Houeland et al., 2010) study, researchers measured the under-expression of NGF in mice; they found that the AD11 mouse exhibited AD-like pathology when there was an under-expression of NGF. Furthermore, these mice also exhibited hippocampal synaptic disfunction (Houeland et al., 2010). NGF under expression and consequent age-dependent appearances of AD-like neurodegeneration can affect synaptic transmission and short-term plasticity of synapses. ${ }^{4}$ These studies have shown that NGF deprivation causes synapse loss in the dentate gyrus, which is a crucial part of the hippocampus. Thus, NGF deprivation could potentially cause the first stages of memory loss in AD. 
Additionally, the "neurotrophic unbalance" hypothesis states that AD-related loss of cholinergic neurons (Ach neurons) and altered APP processing are due to alterations in NGF (Houeland et al., 2010). ${ }^{4}$ These deficiencies include the under expression of NGF by astrocytes, and this leads to neurotoxicity and the degenerative loss of hippocampal neurons. Therefore, alterations in NGF, including the overexpression and under expression, can potentially cause the first stages of $\mathrm{AD}$.

\section{Conclusion}

Astrocytes are crucial elements of the CNS and play an important role in various neurodegenerative diseases, including AD. Additionally, astrocytes have many roles in mediating inflammatory responses and maintaining waste, such as amyloid beta. The imbalance of astrocytes and NGFs can cause various things to happen, such as an increase of inflammation and the production of $\mathrm{A} \beta$.

$\mathrm{AD}$ is one of the most progressive neurodegenerative diseases and could be caused by many factors. For instance, if an imbalance of astrocytes can cause amyloid buildup, and this buildup can cause astrogliosis. Although astrocytes, GF, A $\beta$, and inflammation are very different, they are all part of a complex, dynamic set of interacting processes, affecting all the others. If one element is imbalanced, then it causes a cascade of problems to occur. Targeting astroglia may provide answers for the treatment of AD. Furthermore, targeting astrocytes can help diagnose $\mathrm{AD}$ at an early stage so that treatment is more effective.

\section{Acknowledgements}

I would like to express my gratitude to my research mentor. I thank Andrew Savoy for the guidance and the opportunity to write this paper. Additionally, I would like to thank Horizon for giving me a chance to write my research paper.

\section{References}

[1] Ach, Isacson, O., Seo, H., Lin, L., Albeck, D., Granholm, A., Seo, H., Lin, L., Albeck, D., \& Granholm, A. (n.d.). Helix.mgh.harvard.edu.

[2] Fakhoury, M. (2018). Microglia and Astrocytes in Alzheimer's Disease: Implications for Therapy. Current Neuropharmacology, 16(5), 508-518. https://doi.org/10.2174/1570159X15666170720095240

[3] González-Reyes, R. E., Nava-Mesa, M. O., Vargas-Sánchez, K., Ariza-Salamanca, D., \& Mora-Muñoz, L. (2017). Involvement of Astrocytes in Alzheimer's Disease from a Neuroinflammatory and Oxidative Stress Perspective. Frontiers in Molecular Neuroscience, 10. https://doi.org/10.3389/fnmol.2017.00427

[4] Houeland, G., Romani, A., Marchetti, C., Amato, G., Capsoni, S., Cattaneo, A., \& Marie, H. (2010). Transgenic Mice with Chronic NGF Deprivation and Alzheimer's Disease-Like Pathology Display Hippocampal Region-Specific Impairments in Short- and Long-Term Plasticities. Journal of Neuroscience, 30(39), 13089-13094. https://doi.org/10.1523/JNEUROSCI.0457-10.2010

[5] Ianov, L., De Both, M., Chawla, M. K., Rani, A., Kennedy, A. J., Piras, I., Day, J. J., Siniard, A., Kumar, A., Sweatt, J. D., Barnes, C. A., Huentelman, M. J., \& Foster, T. C. (2017). Hippocampal Transcriptomic Profiles: Subfield Vulnerability to Age and Cognitive Impairment. Frontiers in Aging Neuroscience, 9. https://doi.org/10.3389/fnagi.2017.00383

[6] Nagele, R. G., Wegiel, J., Venkataraman, V., Imaki, H., Wang, K.-C., \& Wegiel, J. (2004). Contribution of glial cells to the development of amyloid plaques in Alzheimer's disease. Neurobiology of Aging, 25(5), 663-674. 
[7] Sajjad, Rabia \& Arif, Rawaba \& Shah, Asad \& Manzoor, Irfan \& Mustafa, Ghulam. (2018). Pathogenesis of Alzheimer's Disease: Role of Amyloid- $\beta$ and Hyperphosphorylated Tau Protein. Indian Journal of Pharmaceutical Sciences. 80. 581-591. 10.4172/pharmaceutical-sciences.1000397.

[8] Tanila, H. (2017). The role of BDNF in Alzheimer's disease. Neurobiology of Disease, 97, 114-118. https://doi.org/10.1016/j.nbd.2016.05.008

[9] Verkhratsky, A., Olabarria, M., Noristani, H. N., Yeh, C.-Y., \& Rodriguez, J. J. (2010). Astrocytes in Alzheimer's disease. Neurotherapeutics, 7(4), 399-412. https://doi.org/10.1016/j.nurt.2010.05.017

[10] (N.d.). Retrieved October 4, 2020, from https://proteinswebteam.github.io/interproblog//potm/2006_7/Page2.htm 